\title{
Transverse and longitudinal components of the propagating and evanescent waves associated to radially-polarized nonparaxial fields
}

\author{
Rosario Martínez-Herrero ${ }^{1}$, Pedro M. Mejías ${ }^{1 *}$, Ignasi Juvells ${ }^{2}$, Artur Carnicer ${ }^{2}$ \\ ${ }^{1}$ Departamento de Óptica, Facultad de Ciencias Físicas \\ Universidad Complutense de Madrid, 28040 Madrid, Spain \\ ${ }^{2}$ Departament de Física Aplicada i Òptica, Facultat de Física, \\ Universitat de Barcelona (UB), 08028 Barcelona, Spain \\ *pmmejias@fis.ucm.es
}

\begin{abstract}
A comparison is established between the contributions of transverse and longitudinal components of both the propagating and the evanescent waves associated to freelypropagating radially-polarized nonparaxial beams. Attention is focused on those fields that remain radially polarized upon propagation. In terms of the plane-wave angular spectrum of these fields, analytical expressions are given for determining both the spatial shape of the above components and their relative weight integrated over the whole transverse plane. The results are applied to two kinds of doughnut-like beams with radial polarization, and compare the behaviour at two transverse planes.
\end{abstract}

\subsection{Introduction}

As is well known, the longitudinal component (along the propagation direction $z$ ) of a light beam is negligible in the paraxial approximation. Consequently, the electric field vector is assumed to be transverse to the z-axis, and represented by means of two components. This provides a considerable simplification in the calculations. However, in a number of applications (for instance, particle trapping, high-density recording and high-resolution microscopy, to mention only some of them), the light beam is strongly focused and raises spot sizes smaller than the wavelength. In such cases, the paraxial approach is no longer valid, and a nonparaxial treatment is required. This is a topic of active research, which has been extensively studied in the last years [1-9]. 
So far, different vectorial formulations of nonparaxial electromagnetic fields have been investigated in the literature (see, for example, [10-13]). Among them, several types of representations based on the plane-wave angular spectrum have been reported in the last years $[5,14,15]$. Such kind of decomposition has revealed to be useful because it allows separate the contribution of the propagating and evanescent waves. In particular, the propagating electric-field solution has been written as the sum of two terms [16]: one of them is transverse to the propagation direction; another one exhibits a non-zero longitudinal component and its associated magnetic field is also transverse. Such analytical description differs from alternative proposals appeared in the literature, also based on the plane-wave spectrum (see [5] and references therein).

In addition, such formalism enables us to analytically incorporate the presence of evanescent waves $[1,5,17,18]$ when their contribution is significant. These waves are receiving increasing attention due, for instance, to their possibilities for subwavelength resolution. As it will be shown in the present paper, the evanescent field can also be written as the sum of transverse and longitudinal parts. We will study and compare the relative weight of all these terms on the basis of the plane-wave angular spectrum of the field. Attention will be focused on beams with radial polarization distributions [2,4,15,1925], a class of fields with important potential applications. More specifically, in the present work we will consider radially polarized beams that retain this character upon free propagation [15]. We refer to them as RPM fields (radially-polarized-maintained fields). Doughnut-like beams with radial polarization represent illustrative examples of these fields (used, for example, as light sources in super-resolution processes).

This paper is organized as follows. In the next section, the formalism and the key definitions are introduced. The field is decomposed as the sum of propagating and evanescent waves. As occurs for the propagation term, the evanescent part split, in turn, into transverse and longitudinal components. Section 3 considers RPM beams, and analyzes the relative contribution of the transverse and longitudinal terms of both the propagating and the evanescent parts. The width of the plane-wave angular spectrum of the field is shown to play a central role in the comparative significance of the longitudinal component of the propagating wave. The above results are applied to several illustrative examples in Section 4. Finally, the main conclusions are summarized in Section 5. 


\section{Formalism and key definitions}

Let us consider a monochromatic electromagnetic beam whose propagation is described by the Maxwell equations (for simplicity, we will consider free-space propagation). As is well known, the electric and magnetic fields, $\boldsymbol{E}$ and $\boldsymbol{H}$, can be expressed in terms of their plane-wave angular spectrum [16],

$$
\begin{aligned}
& \boldsymbol{E}(x, y, z)=\int_{-\infty}^{+\infty} \tilde{\boldsymbol{E}}(u, v, z) \exp [\mathrm{i} k(x u+y v)] d u d v, \\
& \boldsymbol{H}(x, y, z)=\int_{-\infty}^{+\infty} \tilde{\boldsymbol{H}}(u, v, z) \exp [\mathrm{i} k(x u+y v)] d u d v,
\end{aligned}
$$

where $k$ is the wavenumber, and, for simplicity, the time-dependent factor $\exp (-i \omega t)$ has been omitted. In these equations, $\tilde{\boldsymbol{E}}$ and $\tilde{\boldsymbol{H}}$ denote the spatial Fourier transform of $\boldsymbol{E}$ and $\boldsymbol{H}$, respectively. For simplicity, from now on, we will choose $z$ as the direction of propagation of the beam. Instead of using Cartesian coordinates, for the sake of convenience we will next use cylindrical coordinates $R, \theta$ and $z$, i.e.,

$x=R \cos \theta$,

$y=R \sin \theta$,

along with polar coordinates, $\rho$ and $\phi$, related to the transverse Cartesian Fouriertransform variables $u, v$ by the expressions

$u=\rho \cos \phi$,

$v=\rho \sin \phi$.

In terms of these variables, a general solution of the Maxwell equations in the Fourier space can formally be written as follows 
$\tilde{\boldsymbol{E}}(\rho, \phi, z)=\tilde{\boldsymbol{E}}_{0}(\rho, \phi) \exp (i k z \xi)$,

with

$\xi=\left(1-\rho^{2}\right)^{1 / 2}, \quad \rho \leq 1$,

$\xi= \pm \mathrm{i}\left(\rho^{2}-1\right)^{1 / 2}, \quad \rho>1$

where the signs + and - correspond, respectively, to positive $(z>0)$ and negative $(z<0)$ values of the variable $z$. In the present work, attention will be focused on the region $z>0$.

Since the electric field should obey the Maxwell equation $\nabla \cdot \boldsymbol{E}=0$, the Cartesian components of $\tilde{\boldsymbol{E}}_{0}$ should satisfy the condition

$\tilde{E}_{0 x} u+\tilde{E}_{0 y} \nu+\tilde{E}_{0 z} \xi=0$.

The vector $\tilde{\boldsymbol{H}}$ is then obtained from $\tilde{\boldsymbol{E}}_{0}$ in the form

$\tilde{\boldsymbol{H}}(\rho, \phi, z)=\left(\boldsymbol{\sigma} \times \tilde{\boldsymbol{E}}_{0}\right) \exp (i k z \xi)$,

where

$\boldsymbol{\sigma}=(u, v, \xi)=(\rho \cos \phi, \rho \sin \phi, \xi)$,

We see from Eq. (7.b) that the third component of vector $\sigma$ could be a complex number whose presence is closely related with the appearance of evanescent waves (see below).

On the basis of the plane-wave spectrum given by Eq. (4), the electric field solution of the Maxwell equations at any transverse plane $z$ reads

$\boldsymbol{E}(R, \theta, z)=\int_{0}^{\infty} \int_{0}^{2 \pi} \tilde{\boldsymbol{E}}_{0}(\rho, \phi) \exp [\mathrm{i} k \rho R \cos (\theta-\phi)] \exp (\mathrm{i} k z \xi) \rho d \rho d \phi$, 
where $\tilde{\boldsymbol{E}}_{0}$ should fulfil Eq. (5). To our purposes, let us now split this field $\boldsymbol{E}(R, \theta, z)$ as the sum of two terms:

$\boldsymbol{E}(R, \theta, z)=\boldsymbol{E}_{p r}(R, \theta, z)+\boldsymbol{E}_{e v}(R, \theta, z)$,

where

$$
\begin{aligned}
& \boldsymbol{E}_{p r}(R, \theta, z)=\int_{0}^{1} \int_{0}^{2 \pi} \tilde{\boldsymbol{E}}_{0}(\rho, \phi) \exp [\mathrm{i} k \rho R \cos (\theta-\phi)] \exp (\mathrm{i} k z \xi) \rho d \rho d \phi \\
& \boldsymbol{E}_{e v}(R, \theta, z)=\int_{1}^{\infty} \int_{0}^{2 \pi} \widetilde{\boldsymbol{E}}_{0}(\rho, \phi) \exp [\mathrm{i} k \rho R \cos (\theta-\phi)] \exp (\mathrm{i} k z \xi) \rho d \rho d \phi
\end{aligned}
$$

The first term, $\boldsymbol{E}_{p r}$, involves a superposition of plane waves, and represents the contribution of the propagating waves (some authors [26] refer to this term as the homogeneous part of the angular spectrum). The second term of Eq. (9), $\boldsymbol{E}_{\mathrm{ev}}$, should be understood as a superposition of inhomogeneous waves that decay at different rates along the propagation axis. As is well known, close to the initial plane, the evanescent part of the exact solution would be significant enough in the nonparaxial case. Otherwise, $\boldsymbol{E}_{e v}$ is negligible.

Let us now choose a reference system formed by the orthogonal unitary vectors $\boldsymbol{e}_{1}$ and $\boldsymbol{e}_{2}$, namely [16]

$\boldsymbol{e}_{1}=(\sin \phi,-\cos \phi, 0)$,

$\boldsymbol{e}_{2}=\left(\sqrt{1-\rho^{2}} \cos \phi, \sqrt{1-\rho^{2}} \sin \phi,-\rho\right)$

The propagating field can then be written in the form

$$
\boldsymbol{E}_{p r}(\boldsymbol{r})=\int_{0}^{1} \int_{0}^{2 \pi} a(\rho, \phi) \boldsymbol{e}_{1}(\phi) \exp (\mathrm{i} k \boldsymbol{s} \cdot \boldsymbol{r}) \rho d \rho d \phi+\int_{0}^{1} \int_{0}^{2 \pi} b(\rho, \phi) \boldsymbol{e}_{2}(\rho, \phi) \exp (\mathrm{i} k \boldsymbol{s} \cdot \boldsymbol{r}) \rho d \rho d \phi
$$


where functions $a(\rho, \phi)$ and $b(\rho, \phi)$ give the projection of $\widetilde{\boldsymbol{E}}_{0}$ onto the vectors $\boldsymbol{e}_{1}$ and $\boldsymbol{e}_{2}$, respectively, i.e.,

$a(\rho, \phi)=\widetilde{\boldsymbol{E}}_{0} \cdot \boldsymbol{e}_{1}$,

$b(\rho, \phi)=\widetilde{\boldsymbol{E}}_{0} \cdot \boldsymbol{e}_{2}$,

the dot symbolizing the inner product. It is interesting to remark that, for the propagating field $\boldsymbol{E}_{p r}$, condition (5) reduces to

$\tilde{\boldsymbol{E}}_{0}(\rho, \phi) \cdot \boldsymbol{s}(\rho, \phi)=0$,

where

$s(\rho, \phi)=\left(\rho \cos \phi, \rho \sin \phi, \sqrt{1-\rho^{2}}\right)$, with $\rho \in[0,1]$,

is a unitary vector associated to each plan-wave. Thus, the triad $\boldsymbol{s}, \boldsymbol{e}_{1}$ and $\boldsymbol{e}_{2}$ constitute a mutually orthogonal system of unit vectors (see Fig. 1). In fact, the choice of these three vectors characterizes the formalism used in this paper.



Fig. 1 Mutually-orthogonal unitary vectors $\boldsymbol{s}, \boldsymbol{e}_{1}$ and $\boldsymbol{e}_{2}$. Note that vector $\boldsymbol{e}_{1}$ is contained in the transverse plane $\mathrm{x}-\mathrm{y}$ and is orthogonal to the plane formed by vectors $\boldsymbol{e}_{2}$ and $\boldsymbol{s}$, which, in turn, contains the z-axis (remember that these two vectors have longitudinal components along $z$ ). 
Let us finally consider the field solution associated to the evanescent term (cf. Eq. (9) and (10.b)). In a similar way to that used for the propagating term, we can formally write the evanescent part of the field in the form [18]

$\boldsymbol{E}_{e v}=\int_{1}^{\infty} \int_{0}^{2 \pi}\left[a(\rho, \phi) \boldsymbol{e}_{1}(\phi)+b_{e v}(\rho, \phi) \boldsymbol{e}_{e v}(\rho, \phi)\right] \exp \left[\mathrm{i} k\left(\boldsymbol{s}_{e v} \cdot \boldsymbol{r}\right)\right] \rho d \rho d \phi$,

where the unit vector $\boldsymbol{e}_{1}$ was defined before,

$\boldsymbol{e}_{e v}=\frac{1}{\sqrt{2 \rho^{2}-1}}\left( \pm \mathrm{i}\left(\rho^{2}-1\right)^{1 / 2} \cos \phi, \pm \mathrm{i}\left(\rho^{2}-1\right)^{1 / 2} \sin \phi,-\rho\right)$

$\boldsymbol{s}_{e v}=\left(\rho \cos \phi, \rho \sin \phi, \pm \mathrm{i} \sqrt{\left(\rho^{2}-1\right)}\right)$, with $\rho \in[1, \infty]$,

are vectors with complex components (again, the double sign + and - refers to $z>0$ and $z<0$, respectively) and

$b_{\mathrm{ev}}(\rho, \phi)=\tilde{\boldsymbol{E}}_{0} \cdot \boldsymbol{e}_{\mathrm{ev}} \cdot$

In Eq. (18) the product $\boldsymbol{a} \cdot \boldsymbol{b}$ means $a_{x} b_{x}^{*}+a_{y} b_{y}^{*}+a_{z} b_{z}^{*}$. Thus, note that $\boldsymbol{e}_{e v} \cdot \boldsymbol{s}_{e v}=0$ for any $z$. In addition, the field $\boldsymbol{E}_{e v}$ fulfils condition (6) when $\rho \in[1, \infty]$. It should also be remarked that the separate contribution of the evanescent part allows compare the relative weight of this term with regard to the propagating field (by calculating the respective square modulus, integrated throughout the beam profile). This can be used, for example, to find the ranges of both, the propagation distance and the beam size for which the evanescent waves are significant.

This evanescent part can be though as a superposition of inhomogeneous waves whose constant phase surfaces are planes orthogonal to the (non-unitary) transverse vector $\boldsymbol{s}_{0}$, defined in the form

$\boldsymbol{s}_{0}=(\rho \cos \phi, \rho \sin \phi, 0)$, 
and whose constant-amplitude planes are perpendicular to the propagation axis $z$.

The global field $\boldsymbol{E}(R, \theta, z)$ would finally read (see Eq. (9))

$$
\begin{aligned}
& \boldsymbol{E}(R, \theta, z)=\int_{0}^{1} \int_{0}^{2 \pi} a(\rho, \phi) \boldsymbol{e}_{1}(\phi) \exp (i k \boldsymbol{s} \cdot \boldsymbol{r}) \rho d \rho d \phi \\
& +\int_{0}^{1} \int_{0}^{2 \pi} b(\rho, \phi) \boldsymbol{e}_{2}(\rho, \phi) \exp (i k \boldsymbol{s} \cdot \boldsymbol{r}) \rho d \rho d \phi \\
& +\int_{1}^{\infty} \int_{0}^{2 \pi}\left[a(\rho, \phi) \boldsymbol{e}_{1}(\phi)+b_{e v}(\rho, \phi) \boldsymbol{e}_{e v}(\rho, \phi)\right] \exp \left(i k \boldsymbol{s}_{e v} \cdot \boldsymbol{r}\right) \rho d \rho d \phi .
\end{aligned}
$$

It should be noted that the exact solution of the Maxwell equations given by Eq. (20) is valid for both, paraxial and nonparaxial regimes. The angular spectrum $\widetilde{\boldsymbol{E}}_{0}$ at some initial plane would characterize each particular beam.

\section{Radially-polarized-maintained fields}

Let us now consider a general RPM beam. These fields exhibit four main properties, namely,

a) Function $a(\rho, \phi)$ (see Eq. (13.a)) equals zero [15].

b) The plane-wave angular spectrum is independent of $\phi$. This means that $b(\rho, \phi)=b(\rho)$ (see Eq. (13.b)).

c) It can also be shown from Eqs. (12), (17.a) and (18) that $b_{e v}(\rho, \phi)$ does not depend on $\phi$.

d) The longitudinal component differs from zero on the propagation axis $z$ [15].

Taking this behaviour into account, the propagating and evanescent parts of this type of beams become at the initial plane $z=0$

$$
\boldsymbol{E}_{p r}(R, \theta, 0)=\int_{0}^{1} \int_{0}^{2 \pi} b(\rho) \boldsymbol{e}_{2} \exp [\mathrm{i} k \rho R \cos (\theta-\phi)] \rho d \rho d \phi,
$$


and

$\boldsymbol{E}_{e v}(R, \theta, 0)=\int_{1}^{\infty} \int_{0}^{2 \pi} b_{e v}(\rho) \boldsymbol{e}_{e v} \exp [\mathrm{i} k \rho R \cos (\theta-\phi)] \rho d \rho d \phi$.

In particular, the general propagation law of $\boldsymbol{E}_{p r}$ reduces to the simple form [27]

$\boldsymbol{E}_{p r}(R, \theta, z)=\mathrm{i} C_{T}(R, z) \boldsymbol{u}_{\mathrm{R}}+C_{L}(R, z) \boldsymbol{u}_{\mathrm{z}}$,

where $\boldsymbol{u}_{R}=(\cos \theta, \sin \theta, 0)$ and $\boldsymbol{u}_{z}=(0,0,1)$ are unitary vectors in the radial and longitudinal directions, respectively, and

$$
\begin{aligned}
& C_{T}(R, z)=2 \pi \int_{0}^{1} b(\rho) \sqrt{1-\rho^{2}} J_{1}(k R \rho) \exp \left(i k z \sqrt{1-\rho^{2}}\right) \rho d \rho, \\
& C_{L}(R, z)=-2 \pi \int_{0}^{1} \rho b(\rho) J_{0}(k R \rho) \exp \left(i k z \sqrt{1-\rho^{2}}\right) \rho d \rho,
\end{aligned}
$$

$J_{0}$ and $J_{1}$ being the Bessel functions of the first kind of order 0 and 1 , respectively. Since $\boldsymbol{u}_{R}$ is orthogonal to the $z$-axis, the first term of the left-hand side of Eq. (22) would also be both radially polarized and transverse to the propagation axis at any plane $z$.

The polarization state of $\boldsymbol{E}_{p r}$ can be characterized by means of the so-called degree of linear polarization, $\Lambda$, introduced by Lekner in [3]. Thus, in terms of functions $C_{T}(R, z)$ and $C_{L}(R, z)$ given by Eqs. (23.a) and (23.b), we have

$$
\boldsymbol{E}_{p r}^{2}=C_{L}^{2}(R, z)-C_{T}^{2}(R, z)
$$

and

$$
|\boldsymbol{E}|^{2}=\left|C_{L}\right|^{2}+\left|C_{T}\right|^{2}
$$


so that

$\Lambda(R, z)=\frac{\left|C_{L}^{2}-C_{T}^{2}\right|}{\left|C_{L}\right|^{2}+\left|C_{T}\right|^{2}}$.

We see that $\Lambda$ is rotationally symmetric around the $z$-axis (as expected), and the field is no longer linearly polarized after propagation (except along the $z$-axis, where $\Lambda(0, z)=1$ for any $z)$.

The relative contributions of the transverse and longitudinal terms of the propagating field can then be compared each other through the ratio $\left(I_{p r}\right)_{L} /\left(I_{p r}\right)_{T}$, where $\left(I_{p r}\right)_{L}$ and $\left(I_{p r}\right)_{T}$ denote the squared modulus of the longitudinal and transverse parts of $\boldsymbol{E}_{p r}$, integrated over the whole transverse plane. Since the (longitudinal and transverse) components of $\boldsymbol{E}_{p r}$ are written in terms of their plane-wave spectrum, it follows from the application of the Parseval theorem, that $\left(I_{p r}\right)_{L}$ and $\left(I_{p r}\right)_{T}$ do no change upon free propagation. Consequently, it would suffice to calculate the ratio $\left(I_{p r}\right)_{L} /\left(I_{p r}\right)_{T}$ at the initial plane. By taking into account the value of $\boldsymbol{E}_{p r}(R, \theta, 0)$ given by Eq. (21.a), we obtain

$$
\begin{aligned}
& \left(I_{p r}\right)_{T}=2 \pi \int_{0}^{1}|b(\rho)|^{2}\left(1-\rho^{2}\right) \rho d \rho, \\
& \left(I_{p r}\right)_{L}=2 \pi \int_{0}^{1} \rho^{2}|b(\rho)|^{2} \rho d \rho,
\end{aligned}
$$

so that

$$
\frac{\left(I_{p r}\right)_{L}}{\left(I_{p r}\right)_{T}}=\frac{\int_{0}^{1}|b(\rho)|^{2} \rho^{3} d \rho}{\int_{0}^{1}|b(\rho)|^{2}\left(1-\rho^{2}\right) \rho d \rho} .
$$


It is clear from Eqs. (23) and (27) that $C_{T}, C_{L}$ and the ratio $\left(I_{p r}\right)_{L} /\left(I_{p r}\right)_{T}$ are determined by the function $b(\rho)$ (cf. Eq. (13.b)).

To get deeper insight into the physical meaning of function $b(\rho)$, let us consider the ratio (at any $z$ )

$\eta_{z}=\frac{\int_{0}^{\infty} \int_{0}^{2 \pi}\left|\left(E_{p r}\right)_{z}\right|^{2} R d R d \theta}{\int_{0}^{\infty} \int_{0}^{2 \pi}\left|\boldsymbol{E}_{p r}\right|^{2} R d R d \theta}$,

where $\left(E_{p r}\right)_{z}$ denotes the longitudinal component of the propagating wave. In terms of $b(\rho), \eta_{z}$ becomes

$$
\eta_{z}=\frac{\int_{0}^{1} \int_{0}^{2 \pi}|b|^{2} \rho^{3} d \rho d \phi}{\int_{0}^{2 \pi} \int_{0}^{2 \pi}|b|^{2} \rho d \rho d \phi} .
$$

But Eq. (29) can be understood as the (normalized) second-order moment, $\left.<\rho^{2}\right\rangle_{b}$, of function $b(\rho)$. In other words, $\eta_{z}=\left\langle\rho^{2}\right\rangle_{b}$ characterizes the angular size of the region where $|b(\rho)|^{2}$ is important. Consequently, for those RPM fields whose angular spectrum exhibits small values of $\left\langle\rho^{2}\right\rangle_{b}$, the weight of the longitudinal component of the propagating wave would be small, compared with $\left|\boldsymbol{E}_{p r}\right|^{2}$. In the limit $<\rho^{2}>_{b}<<1$, we approach the paraxial regime. On the contrary, high values of $\left\langle\rho^{2}\right\rangle_{b}$ (closed to 1) means that we are considering the nonparaxial case.

With regard to the evanescent wave associated to RPM fields, it can be shown that this wave splits in transverse and longitudinal components, in a similar way to that occurs for the propagating field. Furthermore, the relative weight of such components can be 
compared by using the ratio $\left(I_{e v}\right)_{L} /\left(I_{e v}\right)_{T}$, where $\left(I_{e v}\right)_{L}$ and $\left(I_{e v}\right)_{T}$ represent the squared modulus (integrated over the whole plane) of the longitudinal and transverse components associated to the evanescent part of the field. By using Eq. (21.b), we obtain at plane $z=0$

$$
\begin{aligned}
& \left(I_{e v}\right)_{T}=2 \pi \int_{1}^{\infty}\left|b_{e v}(\rho)\right|^{2} \frac{\rho^{2}-1}{2 \rho^{2}-1} \rho d \rho, \\
& \left(I_{e v}\right)_{l}=2 \pi \int_{1}^{\infty} \rho^{2}\left|b_{e v}(\rho)\right|^{2} \frac{\rho d \rho}{2 \rho^{2}-1},
\end{aligned}
$$

and

$$
\frac{\left(I_{e v}\right)_{L}}{\left(I_{e v}\right)_{T}}=\frac{\int_{1}^{\infty}\left|b_{e v}(\rho)\right|^{2} \frac{\rho^{3}}{2 \rho^{2}-1} d \rho}{\int_{1}^{\infty}\left|b_{e v}(\rho)\right|^{2} \frac{\rho^{2}-1}{2 \rho^{2}-1} \rho d \rho}
$$

In these equations, $\left(I_{e v}\right)_{L}$ and $\left(I_{e v}\right)_{L}$ refer to plane $z=0$. In addition, the ratio $\frac{\left(I_{e v}\right)_{L}}{\left(I_{e v}\right)_{T}}$ can also be written in the form

$$
\frac{\left(I_{e v}\right)_{L}}{\left(I_{e v}\right)_{T}}=1+\delta
$$

where

$$
\delta=\frac{\int_{1}^{\infty} \frac{\left|b_{e v}(\rho)\right|^{2}}{2 \rho^{2}-1} d \rho}{\int_{1}^{\infty}\left|b_{e v}(\rho)\right|^{2} \frac{\rho^{2}-1}{2 \rho^{2}-1} \rho d \rho}>0
$$


$\frac{\left(I_{e v}\right)_{L}}{\left(I_{e v}\right)_{T}}>1$

In other words, for the evanescent term of RPM beams, the contribution of its longitudinal component is always greater than the contribution of the transverse one at any plane transverse to the propagation direction.

\section{Examples}

We will next analyze two kinds of RPM beams characterized by their respective planewave spectrum. Although these fields have been investigated in the literature in recent years, however, to our knowledge, this is the first time in which the behaviour and significance of the transverse and longitudinal components of their associated evanescent waves are studied and compared.

To begin with, let us consider a vectorial distribution $f_{1}(x, y)$ at plane $z=0$ whose angular spectrum $\tilde{f}_{1}$ takes the form [27]

$\tilde{f}_{1}(\rho, \phi)=f(\rho)(0,0,1)$,

where $f(\rho)$ is an scalar function independent of $\phi$. We see from Eq. (34) that the angular spectrum of $\boldsymbol{f}_{1}$ only exhibits a longitudinal component. Unfortunately, this implies that $\boldsymbol{f}_{1}$ does not fulfil the Maxwell equations, and therefore it cannot represent a realistic light field. However, on the basis of the formalism reported in Section 2 (see Eq. (20)), it can be shown that the following field, generated by $\tilde{\boldsymbol{f}}_{1}$, obeys the Maxwell equations:

$$
\begin{aligned}
& \boldsymbol{E}_{1}(R, \theta, z)=\int_{0}^{1} \int_{0}^{2 \pi}\left(\tilde{\boldsymbol{f}}_{1} \cdot \boldsymbol{e}_{2}\right) \boldsymbol{e}_{2}(\rho, \phi) \exp (i k \boldsymbol{s} \cdot \boldsymbol{r}) \rho d \rho d \phi \\
& +\int_{1}^{\infty} \int_{0}^{2 \pi}\left(\tilde{\boldsymbol{f}}_{1} \cdot \boldsymbol{e}_{e v}\right) \boldsymbol{e}_{e v}(\rho, \phi) \exp \left(i k \boldsymbol{s}_{\boldsymbol{e}} \cdot \boldsymbol{r}\right) \rho d \rho d \phi .
\end{aligned}
$$

By comparing this expression with Eq. (20), we obtain 
$a(\rho, \phi)=0 ; b(\rho)=-\rho f(\rho) ; b_{e v}(\rho)=-f(\rho) \frac{\rho}{\sqrt{2 \rho^{2}-1}}$.

The second example is, in a sense, complementary to the first case, because now the starting point is a vector function $\boldsymbol{f}_{2}$ whose plane-wave angular spectrum $\tilde{\boldsymbol{f}}_{2}(\rho, \phi)$ exhibits a radial (transverse) distribution

$\tilde{f}_{2}(\rho, \phi)=f(\rho)(\cos \phi, \sin \phi, 0)$,

with a vanishing longitudinal component. Although $\boldsymbol{f}_{2}$ cannot be accepted as a realistic light field, Eq. (35) is still valid, and the field $\boldsymbol{E}_{2}$ (obtained after substituting $\tilde{\boldsymbol{f}}_{1}$ by $\tilde{\boldsymbol{f}}_{2}$ in this expression) represents another solution of the Maxwell equations. In this case we get (cf. Eq. (20))

$a(\rho, \phi)=0 ; b(\rho)=f(\rho) \sqrt{1-\rho^{2}} ; b_{e v}(\rho)=f(\rho) \frac{i \sqrt{\rho^{2}-1}}{\sqrt{2 \rho^{2}-1}}$

It remains to select function $f(\rho)$ that characterizes the RPM beams chosen in the examples. We will consider an angular spectrum with a doughnut-shape profile of the form

$$
f(\rho)=\frac{\rho}{D} \exp \left(-\frac{\rho^{2}}{D^{2}}\right)
$$

with $D=\frac{1}{k w_{0}}$, where $w_{0}$ denotes a parameter closely connected with the transverse size of the field at the beam waist.

Figure 2 plots the squared modulus of the components of the electric fields $\boldsymbol{E}_{1}$ and $\boldsymbol{E}_{2}$ at the initial plane $z=0$ : Figs. (a) and (b) show the transverse and longitudinal components of the propagating wave, whereas Figs.(c) and (d) refer to the evanescent part. Several properties follow at once from these figures: 
- In the vicinity of the propagation axis $z$, the longitudinal components of both the propagating and the evanescent waves predominate with regard to their respective transverse components. Moreover, the peak value of the longitudinal component of the evanescent wave exceeds the value of the transverse component more than one order of magnitude. This difference is not so strong for the propagating wave.

- Close to the $z$-axis, the magnitudes of the components of the propagating part associated to $\boldsymbol{E}_{1}$ are smaller than the respective components of $\boldsymbol{E}_{2}$.

- The opposite occurs for the evanescent waves. In other words, near the propagation axis, the evanescent part of $\boldsymbol{E}_{1}$ predominates with respect to the evanescent term associated to $\boldsymbol{E}_{2}$.
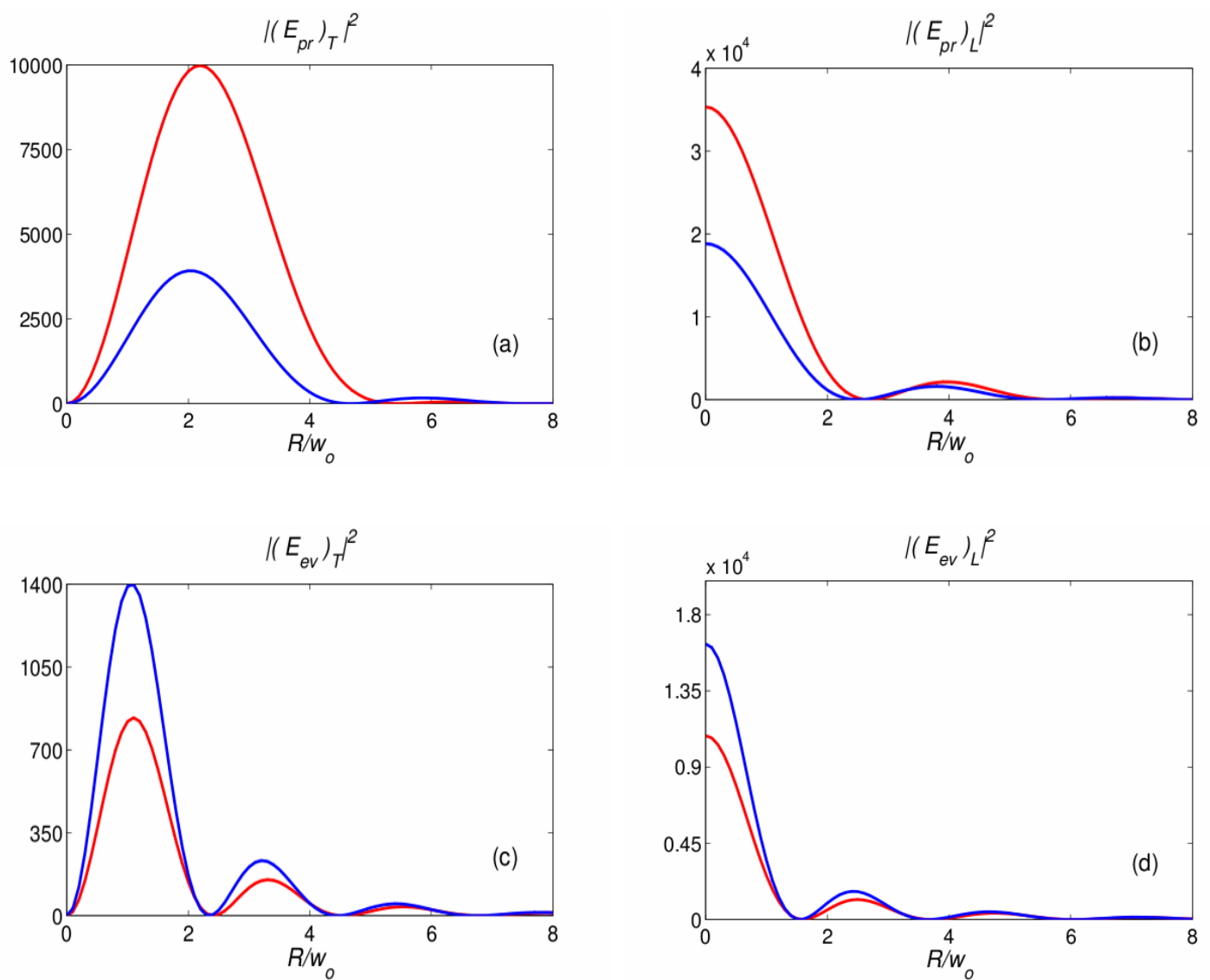

Figure 2. Squared modulus of the field across the initial plane $z=0$ versus the radial distance from the $z$ axis. Note that the beam profiles are rotationally symmetric. In all the figures $w_{0}=0.2 \lambda$. Fig.(a): $\left|\left(\boldsymbol{E}_{p r}\right)_{T}\right|^{2}$; Fig.(b): $\left|\left(\boldsymbol{E}_{p r}\right)_{L}\right|^{2}$; Fig.(c): $\left|\left(\boldsymbol{E}_{e v}\right)_{T}\right|^{2}$; Fig.(d): $\left|\left(\boldsymbol{E}_{e v}\right)_{L}\right|^{2}$. Abscises are given in units of $R / w_{0}$. The numbers that appear in ordinates (arbitrary units) have been given for comparative purposes between the figures (all the curves use the same scale). Blue line refers to the example characterized by Eqs. (34) and (36), and the red line plots the field derived from Eqs. (37) and (38). 
In terms of parameter $w_{0}$, Fig. 3 compares the contributions (i.e., integrated squared modulus) of the transverse and longitudinal components of both the propagating and the evanescent waves at plane $z=0$. From this figure it is apparent that

- The ratio $\left(I_{e v}\right)_{L} /\left(I_{e v}\right)_{T}$ increases almost linearly with $w_{0}$, and takes quite similar values for both fields $\boldsymbol{E}_{1}$ and $\boldsymbol{E}_{2}$. We see that $\left(I_{e v}\right)_{L}$ reaches much higher values than $\left(I_{e v}\right)_{T}$ for wider beams.

- $\quad$ The blue curves (associated to $\boldsymbol{E}_{1}$ ) of Figs. (b)-(d) take higher values than the red curves corresponding to $\boldsymbol{E}_{2}$;

- When $w_{0}$ increases, the curves of Figs. (b)-(d) take lower values. This happens because small values of $w_{0}$ lead to increasing the nonparaxial behaviour. Thus, i) when $w_{0}>0.4 \lambda$, the transverse and the longitudinal components of the evanescent wave become negligible (for both fields $\boldsymbol{E}_{1}$ and $\boldsymbol{E}_{2}$ ), ii) when $w_{0}>\lambda,\left(I_{p r}\right)_{L}<0.1\left(I_{p r}\right)_{T}$ (for both fields $\boldsymbol{E}_{1}$ and $\boldsymbol{E}_{2}$ ).

The above characteristics keep essentially valid after propagating a short distance from the initial plane. Figures 4 and 5 illustrate the behaviour at plane $z=\lambda$. The main qualitative differences with regard to plane $z=0$ can finally be summarized as follows:

- The presence of ripples (circles around the z-axis) in both the transverse and the longitudinal components of the evanescent wave;

- Both fields, $\boldsymbol{E}_{1}$ and $\boldsymbol{E}_{2}$, exhibit quite similar spatial distributions of the components of their respective evanescent terms. This does not happen for the propagating wave in the vicinity of the $z$-axis.

- The contribution of the evanescent wave has significantly reduced, as expected. 



Figure 3. Ratio between transverse and longitudinal components of the propagating and evanescent waves versus the parameter $w_{0}$, which ranges in the interval $[0.2 \lambda, \lambda]$. The curves have been plotted at the initial plane $\mathrm{z}=0$. Blue and red lines have the same meaning as in Fig. 2. Figure (a): $\left(I_{e v}\right)_{L} /\left(I_{e v}\right)_{T}$; Fig. (b): $\left(I_{p r}\right)_{L} /\left(I_{p r}\right)_{T}$; Fig. (c): $\left(I_{e v}\right)_{L} /\left(I_{p r}\right)_{L}$; Fig. (d): $\left(I_{e v}\right)_{T} /\left(I_{p r}\right)_{T}$. Note that the two curves of Fig. (a) are almost coincident. 

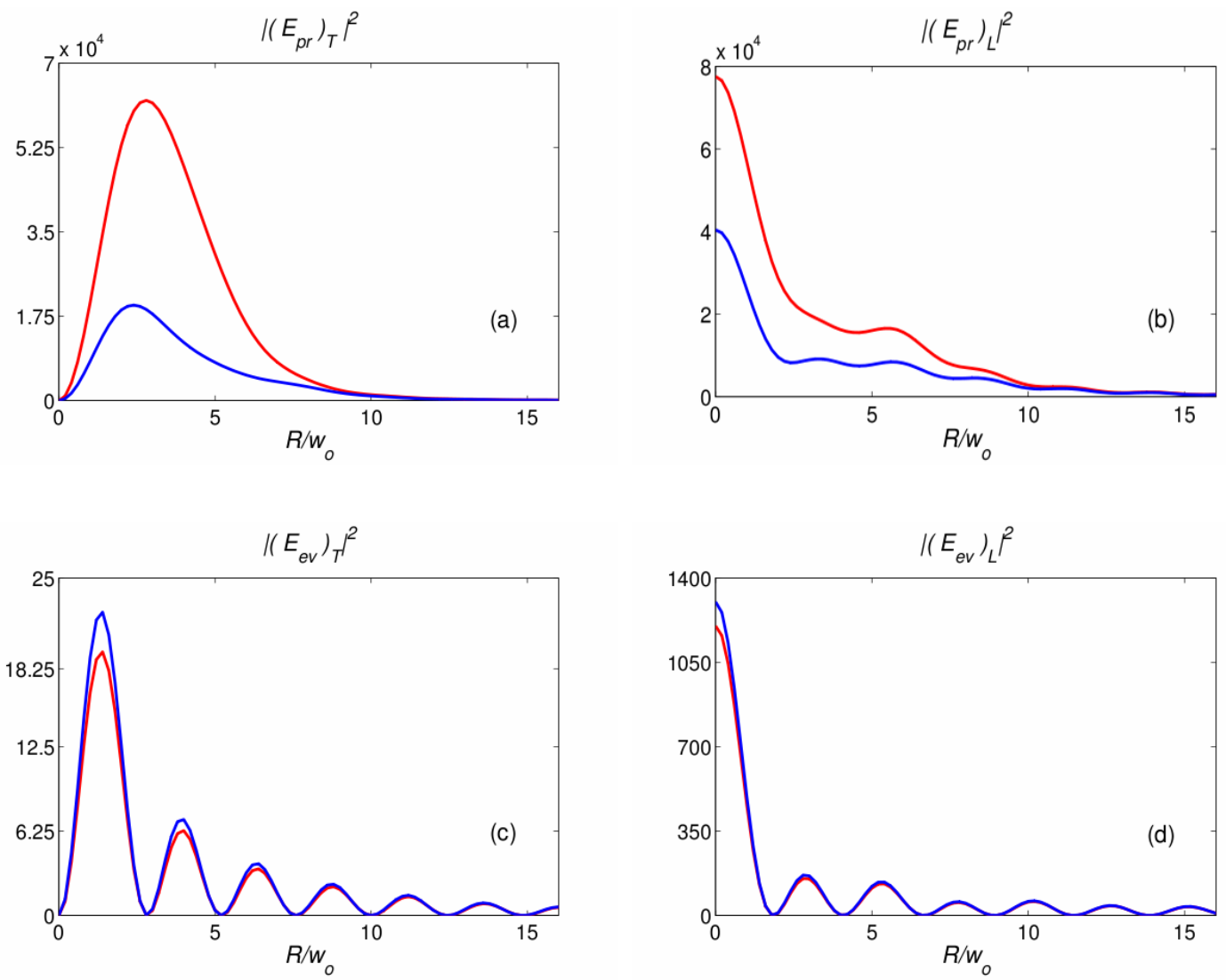

Figure 4. The same as in Fig. 2 but now the curves are plotted at plane $z=\lambda$.
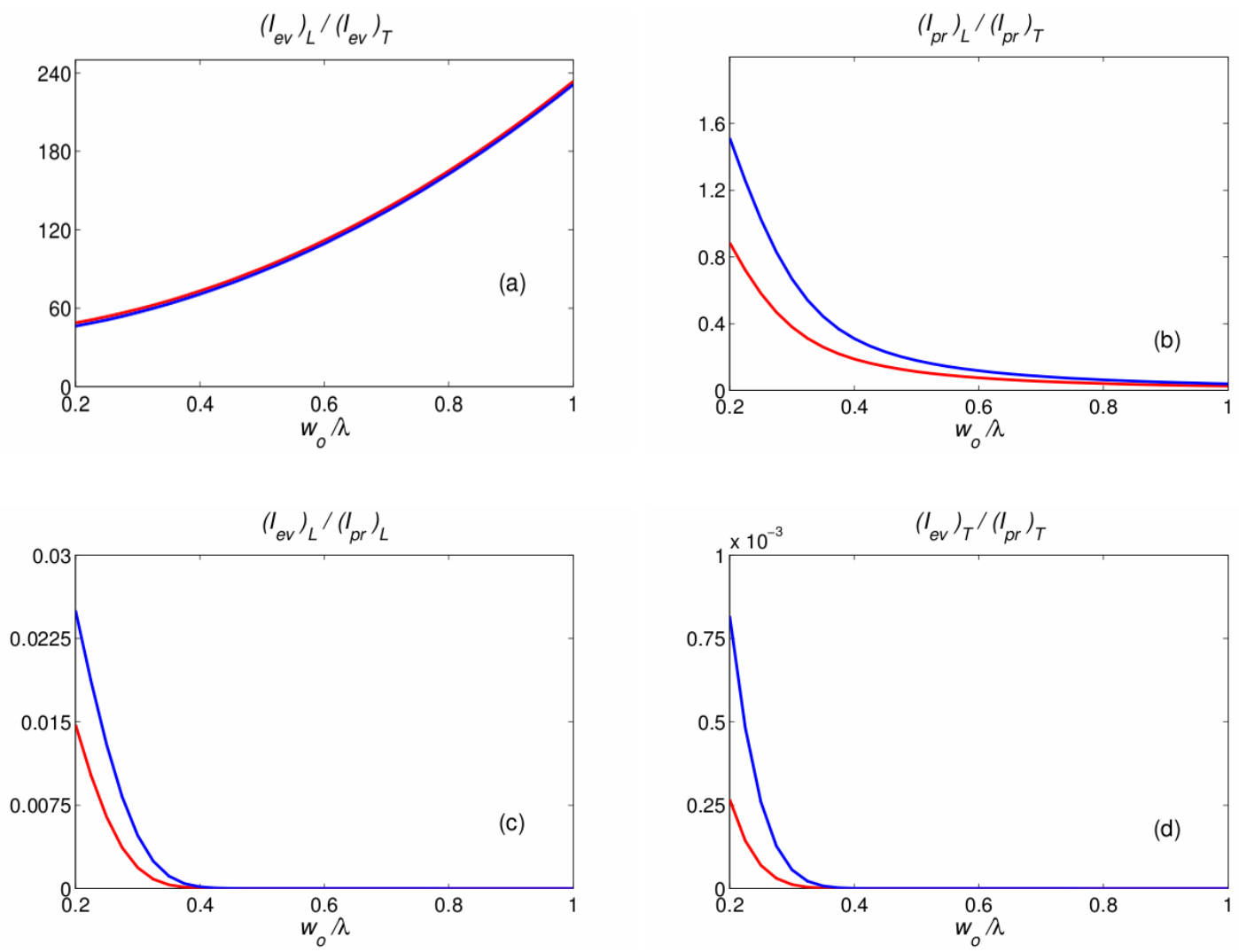

Figure 5. The same as in Fig. 3 but now the curves are plotted at plane $z=\lambda$. 


\section{Conclusions}

In terms of the plane-wave angular spectrum of a light beam, the propagating and the evanescent parts of the nonparaxial field have been split in transverse and longitudinal components. For radially-polarized-maintained beams (i.e., radially polarized fields that retain this character upon propagation), analytical expressions have been provided that allow to compute both the spatial shape of such components and the ratio between their respective contributions, integrated over the whole transverse plane. In addition, this formalism enables to establish the range for which a nonparaxial regime should be considered. In the examples, a comparison has been made between the behavior of two types of doughnut-like beams with radial polarization, along with their evolution under short distance propagation.

This work has been supported by the Ministerio de Ciencia e Innovación of Spain, Project FIS2010-17543.

\section{References}

1. Ciattoni, A., Crosignani, B., Di Porto, P. (2002): Vectorial analytical description of propagation of a highly nonparaxial beam, Opt. Commun. 202, 17-20.

2. Dorn, R., Quabis, S., Lenchs, G. (2003): Sharper focus for a radially polarized light beam, Phys. Rev. Lett. 91, 233901 (1-4).

3. Lekner, J. (2003): Polarization of tightly focused laser beams, J. Opt. A: Pure Appl. Opt. 5, 6-14.

4. Deng, D. M. (2006): Nonparaxial propagation of radially polarized light beams, J. Opt. Soc. Am. B 23, 1228-1234.

5. Chaumet, P. C. (2006): Fully vectorial highly nonparaxial beam close to the waist, J. Opt. Soc. Am. A 23, 3197-3202.

6. April, A. (2008): Nonparaxial TM and TE beams in free space, Opt. Lett. 33, 15631565.

7. Mei, Z. R., Zhao, D. M. (2008): Nonparaxial propagation of controllable dark-hollow beams, J. Opt. Soc. Am A 25, 537-542. 
8. Zhou, G. Q. (2008): The analytical vectorial structure of nonparaxial Gaussian beam close to the source, Opt. Express 16, 3504-3514.

9. Seshadri, S. Q. (2008): Fundamental electromagnetic Gaussian beam beyond the paraxial approximation, J. Opt. Soc Am. A 25, 2156-2164.

10. Varga, P., Török, P. (1996): Exact and approximate solutions of Maxwell's equation and the validity of the scalar wave approximation, Opt. Lett. 21, 1523-1525.

11. Varga, P., Török, P. (1998): The Gaussian wave solution of Maxwell's equations and the validity of the scalar wave approximation, Opt. Commun. 152, 108-118.

12. Sheppard, C. J. R., Saghafi, S. (1999): Electromagnetic Gaussian beams beyond the paraxial approximation, J. Opt. Soc. Am. A 16, 1381-1386.

13. Sheppard, C. J. R. (2000): Polarization of almost-planes waves, J. Opt. Soc. Am. A 17, 335-341.

14. Guo, H. M., Chen, J. B., Zhuang, S. L. (2006): Vector plane wave spectrum of an arbitrary polarized electromagnetic wave, Opt. Express 14, 2095-2100.

15. Martínez-Herrero, R., Mejías, P. M. (2008): Propagation of light fields with radial or azimuthal polarization distribution at a transverse plane, Opt. Express 16, 9021-9033. 16. Martínez-Herrero, R., Mejías, P. M., Bosch, S., Carnicer, A. (2001): Vectorial structure of nonparaxial electromagnetic beams, J. Opt. Soc. Am A, 18, 1678-1680.

17. Petrov, N. I. (1999): Nonparaxial focusing of wave beams in a graded-index medium, Quantum Electron. 29, 249-255.

18. Martínez-Herrero, R., Mejias, P. M., Carnicer, A. (2008): Evanescent field of vectorial highly nonparaxial beams, Opt. Express 16, 2845-2858.

19. Nesterov, A. V., Niziev, V. G., Yakunin, V. P. (1999): Generation of high-power radially polarized beam, J. Phys. D 32, 2871-2875.

20. Niu, C. H., Gu, B. Y., Dong, B. Z., Zhang, Y. (2005): A new method for generating axially-symmetric and radially-polarized beams, J. Phys. D 38, 827-832.

21. Diehl, D. W., Schoonover, R. W., Visser, T. D. (2006): The structure of focused radially polarized fields, Opt. Express 14, 3030-3038.

22. Deng, D. M., Guo, Q. (2007): Analytical vectorial structure of radially polarized light beams, Opt. Lett. 32, 2711-2713.

23. Yew, E. Y. S., Sheppard, C. J. R. (2007): Tight focusing of radially polarized Gaussian and Bessel-Gauss beams, Opt. Lett. 32, 3417-3419.

24. Yan, S. H., Yao, B. L. (2008): Accurate description of a radially polarized Gaussian beam, Phys. Rev. A 77, 023827(1-4). 
25. Machavariani, G., Lumer, Y., Moshe, I., Meir, A., Jackel, S. (2008): Spatiallyvariable retardation plate for efficient generation of radially- and azimuthally-polarized beams, Opt. Commun. 281, 732-738.

26. Sherman, G. C., Stamnes, J. J., Lalor, E. (1976): Asymptotic approximations to angular-spectrum representations, J. Math. Phys. 17, 760-776.

27. Martínez-Herrero, R., Mejías, P. M., Bosch, S. (2008): On the vectorial structure of nonparaxial radially polarized light fields, Opt. Commun. 281, 3046-3050. 